Case Report

\title{
Hypertension Accompanied by Hyperaldosteronism, Hyperkalemia, and Hyperchloremic Acidosis: A Case Report and Literature Review
}

\author{
Yunyun Yang, Yang Ou, Yan Ren, Haoming Tian, and Tao Chen (iD \\ Department of Endocrinology and Metabolism, Adrenal Center, West China Hospital of Sichuan University, Chengdu, \\ Sichuan 610041, China \\ Correspondence should be addressed to Tao Chen; dr.chentao@qq.com
}

Received 4 February 2020; Revised 16 April 2020; Accepted 8 July 2020; Published 24 July 2020

Academic Editor: Toshihiro Kita

Copyright ( 92020 Yunyun Yang et al. This is an open access article distributed under the Creative Commons Attribution License, which permits unrestricted use, distribution, and reproduction in any medium, provided the original work is properly cited.

This study reported on a 24-year-old woman who complained of a paroxysmal headache for six months and elevated blood pressure for four months. Laboratory examination revealed increased serum potassium and chloride levels, metabolic acidosis, suppressed renin activity, and increased plasma aldosterone concentration. Whole-exome sequencing revealed a heterozygous mutation in exon 11 of the KLHL3 gene: c.1298G > A. After treatment with low-dose hydrochlorothiazide, her clinical problems were controlled. This patient is the first case of Gordon syndrome (GS) within the Chinese population caused by a heterozygous KLHL3 mutation. A systematic review of the published literature identified 27 patients with GS caused by a KLHL3 mutation. These patients had a mean age of $28.2 \pm 22.0$ years; $74.1 \%$ presented with hypertension, $76.9 \%$ with hyperkalemia, and $59.1 \%$ with metabolic acidosis. The patients also had varying levels of plasma renin activity and plasma aldosterone concentrations.

\section{Introduction}

Gordon syndrome (GS), also known as familial hyperkalemic hypertension or pseudohypoaldosteronism type II (PHA II), is a rare type of familial hypertension. GS results from excessive activation of the thiazide-sensitive $\mathrm{Na} / \mathrm{Cl}$ cotransporter (NCC) in the distal convoluted tubules, which leads to an increase in $\mathrm{NaCl}$ reabsorption and decreases in $\mathrm{K}^{+}$and $\mathrm{H}^{+}$excretion, eventually resulting in high blood pressure, hyperkalemia, hyperchloremic metabolic acidosis, suppressed renin levels, and variable aldosterone levels. Other clinical manifestations of GS include growth disorders, myasthenia, periodic paralysis, skeletal abnormalities, and psychomotor delay [1]. Mutations in at least four genes are responsible for GS, including the WNK1, WNK4, KLHL3, and CUL3 genes. A large cohort study of PHA II kindreds illustrated that mutations in KLHL3 and CUL3 were the leading causes (approximately 79\%), and mutations in WNK1 or WNK4 only accounted for a small number of PHAII kindreds (approximately 13\%).
The present case was a 24-year-old young woman with hypertension, hyperkalemia, hyperchloremia, metabolic acidosis, low renin, and elevated aldosterone. Whole-exome sequencing of the patient revealed a KLHL3 heterozygous mutation: c.1298G > A. After treatment with low-dose hydrochlorothiazide, the patient's high blood pressure, hyperkalemia, and hyperchloremic metabolic acidosis gradually normalized. In addition, the present study systematically reviewed the published literature about the Gordon syndrome with KLHL3 mutations and summarized the clinical characteristics of genetically confirmed GS cases.

\section{Case Presentation}

The patient was a 24-year-old young woman complaining of a paroxysmal headache for six months and elevated blood pressure for four months. Six months ago, she experienced an episodic headache after sleep insufficiency. Four months ago, she was found with hypertension (up to 150/ $109 \mathrm{mmHg}$ ) in a health examination. The patient had regular 
menstruation. Her father, grandfather, and grandmother had hypertension. Physical examination of this patient showed no abnormal signs except elevated blood pressure. As shown in Table 1, the patient had elevated levels of serum potassium and chloride, decreased arterial blood $\mathrm{pH}$, standard bicarbonate concentration, and normal serum creatinine and glomerular filtration rate. The patient had suppressed plasma renin activity, elevated plasma aldosterone concentration, normal cortisol circadian rhythm and levels, and normal levels of 24-hour urinary free cortisol, adrenocorticotropin, and serum catecholamine. Moreover, the captopril challenge test of the patient suggested that her plasma aldosterone was suppressed by $31.6 \%$. Her sex hormones, thyroid hormone, and parathyroid hormone were normal. Magnetic resonance imaging (MRI) examination suggested no obvious abnormality in the bilateral adrenal glands. Ultrasound of the urinary system indicated uric acid crystals in the right kidney.

The patient's father had hypertension, and her mother's blood pressure was normal; both were unavailable for laboratory examination and genetic screening. Her elder brother had normal blood pressure, normal levels of serum potassium and serum chlorine, and normal arterial blood gas analysis results.

The peripheral blood genomic DNA of the patient and her elder brother were extracted (Tiangen DNA extraction kit). Next-generation sequencing was applied to capture suspicious mutation sites in the index patient (Illumina HiSeq 2500 system). The suspected mutation was confirmed by direct sequencing of the index patient and other available individuals (Beijing Zhiyin Oriental Transformational Medicine Research Center Co., Ltd.). The patient was identified with a heterozygous mutation in exon 11 of the KLHL3 gene, c.1298G $>$ A, which led to $433^{\text {rd }}$ amino acid serine to be replaced by asparagine (S433N). Her elder brother was wild type at this site (Figure 1). According to the genetic findings, as well as typical clinical manifestations, the patient was diagnosed with the Gordon syndrome.

After treatment with low-dose hydrochlorothiazide ( $25 \mathrm{mg} /$ day), her blood pressure fluctuated in the range of 90-100/70-80 $\mathrm{mmHg}$, her blood potassium and chlorine, plasma renin activity, and aldosterone concentration returned to normal, and metabolic acidosis was corrected (Table 1). The patient continued to take the low-dose hydrochlorothiazide treatment after discharge. Her blood pressure and serum potassium were normal three months later at follow-up.

\section{Review of Clinical Features and Laboratory Findings of GS Patients}

To comprehensively review the clinical characteristics of GS caused by the KLHL3 gene mutation, we searched for primary studies published through December 2019 on MEDLINE (PubMed) and the Chinese Biomedical Database (CBM). The MeSH terms "Gordon syndrome," "Pseudohypoaldosteronism type II," and "familial hyperkalemic hypertension" were used for the literature search. The retrieved articles in English and Chinese languages were independently screened by two authors (YY Yang and T Chen). Full-text case studies were included only if they had available data on clinical features and laboratory findings. Clinical data, including country of origin, age at symptom onset, gender, and clinical manifestations such as blood pressure, serum potassium, arterial $\mathrm{pH}$ value, plasma renin activity (PRA)/concentration (PRC), and plasma aldosterone concentration (PAC), were sorted out and analyzed.

We identified 27 studies in total, then excluded 22 studies that lacked genetic tests or detailed clinical manifestations or that had mutations other than those in the KLHL3 gene, and eventually included five studies that contained 27 affected members from 8 pedigrees (Table 2). No studies in the Chinese language about the KLHL3 gene mutation were retrieved. The mean age at diagnosis was $28.2 \pm 22.0$ years, and $59.3 \%$ of the patients were females. The incidences of hypertension, hyperkalemia, and metabolic acidosis were $74.1 \%, 76.9 \%$, and $59.1 \%$, respectively. Among GS patients with hypertension $(n=20), 45 \%$ of them were diagnosed with hypertension before the age of 18 years, and the overall median age was 27.6 years (range: $0.8-62$ years). Among GS patients with hyperkalemia $(n=20)$, the median age was 21.6 years. Among GS patients with metabolic acidosis $(n=13)$, the median age was 22.7 years. In addition, 73.9\% of GS patients presented with suppressed PRA, 26.1\% presented with normal PRA, 38.1\% presented with low PAC, $52.4 \%$ presented with normal PAC, and $9.5 \%$ presented with high PAC.

\section{Discussion}

This study reported on a 24-year-old patient who presented with elevated blood pressure and increased serum potassium and chlorine, as well as decreased arterial blood $\mathrm{pH}$, standard bicarbonate concentration, suppressed PRA, normal serum creatinine level, and normal estimated glomerular filtration rate. The above clinical clues indicated a possible diagnosis of the Gordon syndrome. Whole-exome sequencing revealed that the patient carried a heterozygous mutation in the KLHL3 gene: c.1298G > A, which led to $433^{\text {rd }}$ amino acid serine to be replaced by asparagine (S433N). The patient's elder brother, who had normal blood pressure and normal serum potassium, was wild type at this site.

KLHL3 along with the WNK1, WNK4, and CUL3 genes are involved in the physiologic regulation of NCC transporter activity [1,7-10]. Mutations in the WNK1, WNK4, KLHL3, and CUL3 genes lead to increased NCC transporter activity and membrane expression $[7,11,12]$, enhance the inhibition of ROMK $[7,9,11]$, and eventually lead to typical clinical features of GS. Whole-exome sequencing in this patient revealed a heterozygous mutation in exon 11 of KLHL3 (c.1298G > A). This mutation was first reported in a large cohort study of 52 European and American GS kindreds in 2012 [8]. Then, a 2017 study from South Korea reported two cases of GS carrying the same mutation, which included a 25-month-old female infant and her 30-year-old mother. The daughter had hypertension, hyperkalemia $(6.9 \mathrm{mmol} / \mathrm{L})$, and metabolic acidosis [13], and the mother 
TABLE 1: Laboratory findings of the index patient.

\begin{tabular}{lccc}
\hline Biochemical findings & Pre-HCTZ & Post-HCTZ & Reference values \\
\hline Serum potassium & 6.3 & 4.00 & $3.5-5.3 \mathrm{mmol} / \mathrm{L}$ \\
Serum sodium & 142.8 & 138.9 & $137-147 \mathrm{mmol} / \mathrm{L}$ \\
Serum chlorine & 114.7 & 96.4 & $99-110 \mathrm{mmol} / \mathrm{L}$ \\
Serum creatinine & 60 & N/A & $37-110 \mu \mathrm{mol} / \mathrm{L}$ \\
eGFR & 122 & N/A & $56-122 \mathrm{~mL} / \mathrm{min}^{2} / 1.73 \mathrm{~m}$ \\
Arterial blood pH value & 7.286 & 7.400 & $7.35-7.45$ \\
$\mathrm{HCO}_{3}^{-}$ & 17.6 & 26.2 & $22-27 \mathrm{mmol} / \mathrm{L}$ \\
PRA renin activity & 0.14 & 1.07 & $0.93-6.56 \mathrm{ng} / \mathrm{mL} / \mathrm{h}$ \\
PRC aldosterone & 31.00 & 15.2 & $9.8-27.6 \mathrm{ng} / \mathrm{dl}$ \\
Serum cortisol (8 AM) & 300.2 & N/A & $147.3-609.3 \mathrm{nmol} / \mathrm{L}$ \\
Serum cortisol (4 PM) & 162.0 & N/A & $34-340 \mathrm{nmol} / \mathrm{L}$ \\
Serum cortisol (midnight) & 57.2 & N/A & $<50 \mathrm{nmol} / \mathrm{L}$ \\
24-h UFC & 43.61 & N/A & $20.3-127.6 \mu \mathrm{g} / 24 \mathrm{~h}$ \\
ACTH & 21.53 & N/A & $5.0-78 \mathrm{ng} / \mathrm{L}$ \\
\hline
\end{tabular}

Note. eGFR: estimated glomerular filtration rate; ACTH: adrenocorticotropin; UFC: urinary free cortisol; $\mathrm{HCO}_{3}{ }^{-}$: serum bicarbonate; PRA: plasma renin activity; PRC: plasma aldosterone concentration; and HCTZ: hydrochlorothiazide.

- KLHL3: c. 1298 (exon11) G>A:

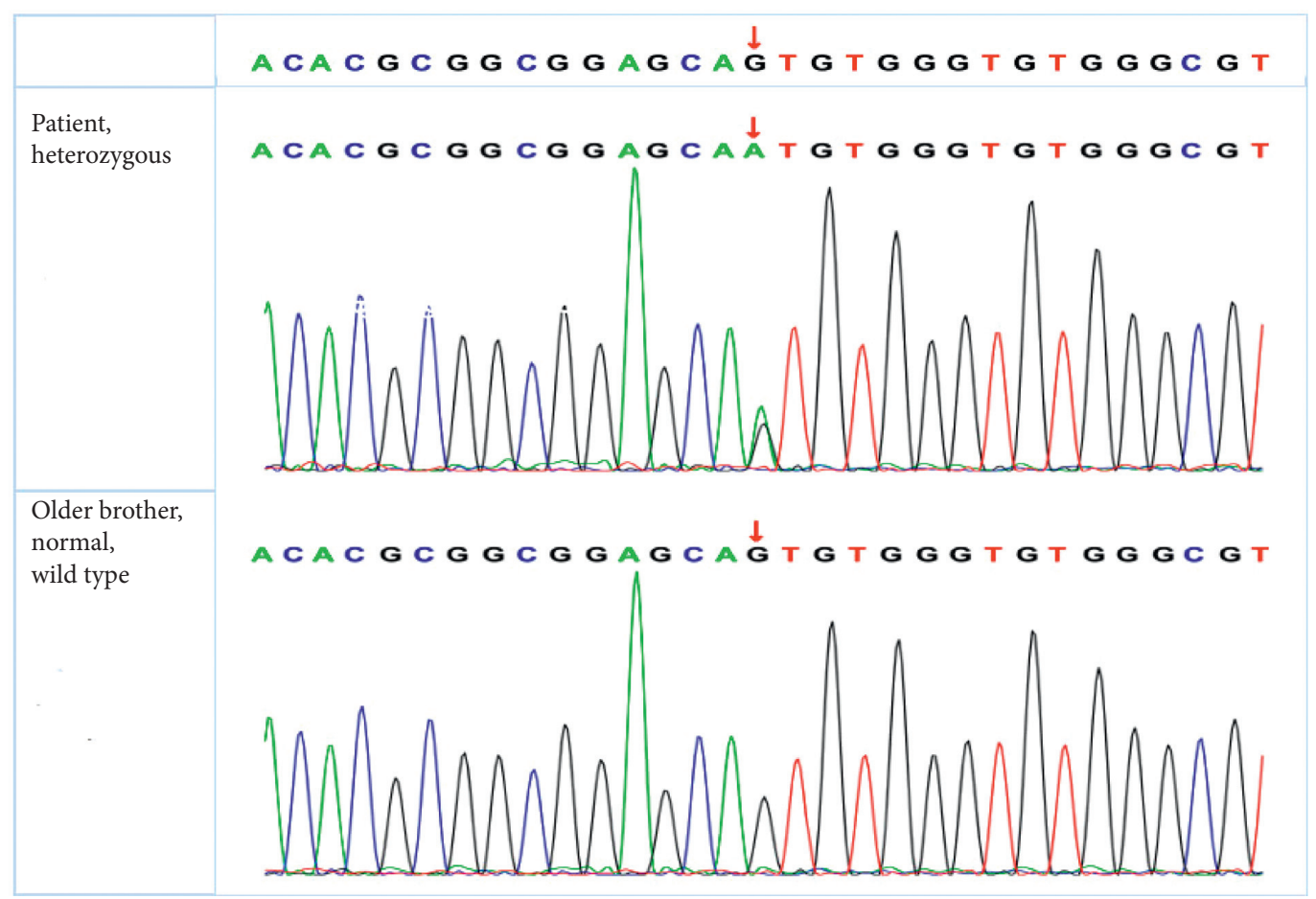

Figure 1: Whole-exome sequencing showing a novel KLHL3 mutation.

had hypertension and hyperkalemia in her twenties [4]. Previously reported GS cases from the Chinese population were mostly caused by WNK4 mutations and CUL3 mutations [14]. To date, no study has reported GS cases with KLHL3 dominant mutations among the Chinese population.

There are significant differences in the severity of clinical phenotypes among GS patients with different gene mutations. CUL3 gene mutation tends to result in much more severe clinical phenotype followed by KLHL3, WNK4, and WNK1 gene mutations. Patients with CUL3 mutations usually developed symptoms at younger ages, with more severe hyperkalemia and metabolic acidosis $[7,8]$, while patients with KLHL3 mutations usually developed symptoms after adulthood, with mild-to-moderate clinical manifestations.

This patient presented typical features of GS, e.g., hypertension, hyperkalemia, hyperchloremia, and metabolic acidosis, while the systematic review of published studies showed that the clinical manifestations varied among GS patients; hyperkalemia was the most common clinical symptom, which was present in $76.9 \%$ of GS patients followed by hypertension (74.1\%) and metabolic acidosis (59.1\%). Compared with hyperkalemia and metabolic acidosis, hypertension seemed to develop at a later stage 
TABLE 2: Systematic review of clinical features and laboratory findings of GS patients with KLHL3 gene mutations.

\begin{tabular}{|c|c|c|c|c|c|c|c|c|c|}
\hline ID & Mutation & Age (years) & Sex & $\mathrm{BP}$ & $\mathrm{K}^{+}$ & $\mathrm{pH}$ & $\mathrm{HCO}_{3}{ }^{-}$ & PRA & PAC \\
\hline Kelly et al. [2] & H498Y & 18 & M & $150 / 90$ & 7.3 & 7.34 & 18 & 0.2 & 19.1 \\
\hline Mitani et al. [3] & L387P & 3 & $\mathrm{M}$ & $110 / 55$ & 6.6 & 7.34 & 14.8 & 0.6 & 6.6 \\
\hline \multirow{3}{*}{ Park et al. [4] } & $\mathrm{C} 164 \mathrm{~F}$ & 0.8 & $\mathrm{~F}$ & $115 / 72$ & 6.3 & 7.34 & 19.6 & $<0.1$ & 6.4 \\
\hline & S433N & 1.7 & $\mathrm{~F}$ & $110 / 73$ & 6.9 & 7.30 & 12 & $<0.1$ & 7.1 \\
\hline & S433N & 23 & $\mathrm{~F}$ & Hypertension & 6.4 & NA & NA & NA & NA \\
\hline \multirow{10}{*}{ Mayan et al. [5] } & Q309R & 69 & $\mathrm{~F}$ & $115 / 78$ & 5.4 & NA & 27 & 2.3 & 10.6 \\
\hline & Q309R & 48 & $\mathrm{~F}$ & $147 / 95$ & 5.2 & NA & 25.7 & 0.1 & 4.1 \\
\hline & Q309R & 11 & $\mathrm{M}$ & $104 / 61$ & 6.4 & NA & 22.3 & 0.1 & 23.8 \\
\hline & Q309R & 11 & $\mathrm{M}$ & $108 / 71$ & 7.2 & NA & 21.5 & 0.1 & 14.0 \\
\hline & Q309R & 10 & $\mathrm{~F}$ & $103 / 72$ & 5.3 & NA & 22.7 & 0.7 & 23.9 \\
\hline & $\mathrm{R} 528 \mathrm{H}$ & 38 & $\mathrm{~F}$ & $154 / 77$ & 5.6 & NA & 18 & 3.6 & 51.5 \\
\hline & $\mathrm{R} 528 \mathrm{H}$ & 15 & $\mathrm{~F}$ & $136 / 77$ & 6.0 & NA & 19.7 & 0.3 & 4.1 \\
\hline & $\mathrm{R} 528 \mathrm{H}$ & 13 & $\mathrm{~F}$ & $124 / 77$ & 6.2 & NA & 19.8 & $<0.3$ & 14.8 \\
\hline & $\mathrm{R} 528 \mathrm{H}$ & 8 & $\mathrm{~F}$ & $123 / 78$ & 5.8 & NA & 19.1 & 0.7 & 10.7 \\
\hline & $\mathrm{R} 528 \mathrm{H}$ & 2 & M & $146 / 95$ & 7.0 & NA & NA & 0.3 & 28.3 \\
\hline \multirow{12}{*}{ Kliuk et al. [6] } & S553L & 34 & $\mathrm{~F}$ & $158 / 107$ & 6.2 & NA & NA & $<0.5$ & 15.5 \\
\hline & S553L & 26 & M & $115 / 70$ & 7.1 & NA & NA & $<0.5$ & 6.5 \\
\hline & Q309R & 63 & $\mathrm{M}$ & $110 / 80$ & 5.6 & NA & 19.0 & 1.4 & 4.5 \\
\hline & Q309R & 62 & $\mathrm{~F}$ & $160 / 100$ & 4.8 & NA & 23.6 & 1.7 & 8 \\
\hline & Q309R & 57 & $\mathrm{M}$ & $120 / 90$ & 5.1 & NA & 19.7 & NA & 16.7 \\
\hline & Q309R & 54 & $\mathrm{M}$ & $145 / 105$ & 6.4 & NA & 23.1 & 4.7 & 24.6 \\
\hline & Q309R & 47 & $\mathrm{~F}$ & $120 / 90$ & 5.1 & NA & 23.8 & 2.5 & 21.8 \\
\hline & Q309R & 47 & $\mathrm{~F}$ & $145 / 110$ & 5.1 & NA & 19.5 & $<1.0$ & NA \\
\hline & Q309R & 44 & $\mathrm{M}$ & $160 / 100$ & NA & NA & NA & NA & NA \\
\hline & Q309R & 18 & $\mathrm{M}$ & $110 / 70$ & 5.2 & NA & 23.9 & $<0.5$ & NA \\
\hline & Q309R & 15 & $\mathrm{~F}$ & $110 / 80$ & 5.8 & NA & 20.2 & $<0.5$ & NA \\
\hline & Q309R & 12 & $\mathrm{~F}$ & $114 / 76$ & 5.3 & NA & 22 & NA & NA \\
\hline Present case & S433N & 24 & $\mathrm{~F}$ & $150 / 109$ & 6.3 & 7.286 & 17.6 & 0.14 & 31.0 \\
\hline
\end{tabular}

(median age: 27.6 versus 21.6 and 22.7 years). This evidence indicated that GS should be suspected when young patients present with hyperkalemia, even those who do not present with hypertension and metabolic acidosis.

Most of the previous studies reported that GS patients had suppressed renin activity but low or normal plasma aldosterone concentrations [2, 4, 14-17]. For instance, one of the South Korean cases of GS caused by the same mutation in the present case (S433N) had a suppressed RAAS (plasma renin activity less than $0.10 \mathrm{ng} / \mathrm{mL} / \mathrm{h}$ and plasma aldosterone $7.1 \mathrm{ng} / \mathrm{dL})$. Similarly, patients with other sites of KLHL3 mutations (such as Q309R, R528H, H498Y, and L387P) had low plasma renin activity and normal or decreased aldosterone levels [2-5]. The possible mechanism may be associated with increased NCC activity, which leads to increased $\mathrm{NaCl}$ reabsorption and volume-dependent hypertension, thus inhibiting the RAAS (decreased renin activity and aldosterone levels).

Interestingly, the present case presented with suppressed PRA and slightly increased PAC. Similarly, as shown in the results of the literature review and other published studies, there were also some cases or pedigrees with GS characterized by an elevated PAC, which was mainly caused by WNK4 or CUL3 mutations [18-20]. The possible mechanism could be related to the regulatory effects of hyperkalemia on aldosterone secretion. For example, one case with a WNK4 mutation had a potassium level of $6.4 \mathrm{mmol} / \mathrm{L}$ with a corresponding PAC of $50.7 \mathrm{ng} / \mathrm{dL}$, while when serum potassium decreased to $5.4 \mathrm{mmol} / \mathrm{L}$, the corresponding PAC decreased to $13.7 \mathrm{ng} / \mathrm{dL}$ [19]. This phenomenon could also be observed in our patient, whose PAC was $31.0 \mathrm{ng} / \mathrm{dL}$ when serum potassium was $6.3 \mathrm{mmol} / \mathrm{L}$, which decreased to $15.2 \mathrm{ng} / \mathrm{dL}$ when serum potassium decreased to $4.9 \mathrm{mmol} / \mathrm{L}$. In brief, the above evidence suggested that the RAAS among GS patients is characterized by a suppressed PRA but variable PAC that is positively associated with serum potassium level. Moreover, a suppressed PRA will lead to an increased aldosterone/renin ratio (ARR), which, in addition to elevated blood pressure, might result in inappropriate screening for primary aldosteronism.

GS patients respond to low doses of thiazide diuretics by inhibition of the activity of NCC in the distal nephrons. Previous studies have reported that thiazide diuretics could correct the clinical manifestations, including hypertension, hyperkalemia, and metabolic acidosis, in almost all GS patients [7, 8]. The present patient accepted treatment of low-dose hydrochlorothiazide ( $25 \mathrm{mg} /$ day). Her elevated blood pressure, serum potassium, and metabolic acidosis were corrected five days later and maintained at normal levels at three months of follow-up.

\section{Conclusions}

This study reported on a case of the Gordon syndrome with a heterozygous mutation (c.1298G $>$ A) in exon 11 of the KLHL3 gene. The patient had typical features of the Gordon 
syndrome and responded well to low-dose thiazide diuretics. GS patients with KLHL3 gene mutations present with varied clinical manifestations and might be confused with primary aldosteronism.

\section{Abbreviations}

GS: $\quad$ Gordon syndrome

PHA II: Pseudohypoaldosteronism type II

FHH: Familial hyperkalemic hypertension

WNK: With-no-lysine kinase

KLHL3: Kelch-like 3

CUL3: Cullin 3

OSR1: Oxidative stress-responsive gene 1

SPAK: STE20/SPS1-related proline-alanine-rich kinase

CRL: Cullin-RING E3 ubiquitin ligase

DCT: Distal convoluted tubules

NCC: $\mathrm{Na} / \mathrm{Cl}$ cotransporter

ENaC: Epithelial sodium $(\mathrm{Na}+)$ channel

ROMK: Renal outer medullary potassium $(\mathrm{K}+)$ channel

RAAS: Renin-angiotensin-aldosterone system

PRA: Plasma renin activity

PAC: Plasma aldosterone concentration

AM: $\quad$ Acidic motif.

\section{Ethical Approval}

All procedures performed in studies involving human participants followed the ethical standards of the Human Ethics Committees of the West China Hospital of Sichuan University (2016/323) and the 1964 Helsinki Declaration and its later amendments or comparable ethical standards.

\section{Consent}

Informed consent was obtained from the patient and family members.

\section{Conflicts of Interest}

The authors declare that they have no conflicts of interest.

\section{Authors' Contributions}

TC conceived the study and participated in its design and coordination. YYY collected the clinical information of the participants and drafted the manuscript. YR collected the clinical information of the participants. HMT edited the manuscript. All authors read and approved the final manuscript.

\section{Acknowledgments}

This study was supported by grants from a 1.3.5 Project for Disciplines of Excellence, West China Hospital, Sichuan University (ZYGD18022 and 2018HXFH009) and a grant from the Sichuan Provincial Science and Technology Foundation (2019YJ0040).

\section{References}

[1] D. H. Ellison, "Pseudohypoaldosteronism type II," in Gene Reviews, R. A. Paqon, M. P. Adam, and H. H. Ardinqer, Eds., University of Washington, Seattle, WA, USA, 2017, http:// www.genereviews.org.

[2] D. Kelly, M. R. Rodzlan, X. Jeunemaitre, and C. Wall, “A novel mutation in KLHL3 gene causes familial hyperkalemic hypertension," QJM: An International Journal of Medicine, vol. 109, no. 7, pp. 487-488, 2016.

[3] M. Mitani, M. Furuichi, S. Narumi et al., "A patient with pseudohypoaldosteronism type II complicated by congenital hypopituitarism carrying a KLHL3 mutation," Clinical Pediatric Endocrinology, vol. 25, no. 4, pp. 127-134, 2016.

[4] J. S. Park, E. Park, H. S. Hyun et al., "Three cases of Gordon syndrome with dominant KLHL3 mutations," Journal of Pediatric Endocrinology and Metabolism, vol. 30, no. 3, pp. 361-364, 2017.

[5] H. Mayan, V. Carmon, K. Oleinikov et al., "Hypercalciuria in familial hyperkalemia and hypertension with KLHL3 mutations," Nephron, vol. 130, no. 1, pp. 59-65, 2015.

[6] O. Kliuk-Ben Bassat, V. Carmon, A. Hanukoglu et al., "Familial hyperkalemia and hypertension (FHHt) and KLHL3: description of a family with a new recessive mutation (S553L) compared to a family with a dominant mutation, Q309R, with analysis of urinary sodium chloride cotransporter," Nephron, vol. 137 , no. 1 , pp. 77-84, 2017.

[7] K. M. O Shaughnessy, "Gordon Syndrome: a continuing story," Pediatric Nephrology, vol. 30, no. 11, pp. 1903-1908, 2015.

[8] L. M. Boyden, M. Choi, K. A. Choate et al., "Mutations in kelch-like 3 and cullin 3 cause hypertension and electrolyte abnormalities," Nature, vol. 482, no. 7383, pp. 98-102, 2012.

[9] J. K. Healy, "Pseudohypoaldosteronism type II," Hypertension, vol. 63, no. 4, pp. 648-654, 2014.

[10] L. Wang and J.-B. Peng, "Phosphorylation of KLHL3 at serine 433 impairs its interaction with the acidic motif of WNK4: a molecular dynamics study," Protein Science, vol. 26, no. 2, pp. 163-173, 2017.

[11] E. Sohara and S. Uchida, "Kelch-like 3/Cullin 3 ubiquitin ligase complex and WNK signaling in salt-sensitive hypertension and electrolyte disorder," Nephrology Dialysis Transplantation, vol. 31, no. 9, pp. 1417-1424, 2016.

[12] T. Moriguchi, S. Urushiyama, N. Hisamoto et al., "WNK1 regulates phosphorylation of cation-chloride-coupled cotransporters via the STE20-related kinases, SPAK and OSR1," Journal of Biological Chemistry, vol. 280, no. 52, pp. 42685-42693, 2005.

[13] M. Guzman-Limon and J. Samuels, "Pediatric hypertension," Pediatric Clinics of North America, vol. 66, no. 1, pp. 45-57, 2019.

[14] L. Shao, L. Cui, J. Lu, Y. Lang, I. Bottillo, and X. Zhao, “A novel mutation in exon 9 of Cullin 3 gene contributes to aberrant splicing in pseudohypoaldosteronism type II," FEBS Open Bio, vol. 8, no. 3, pp. 461-469, 2018.

[15] H. Gong, Z. Tang, Y. Yang et al., "A patient with pseudohypoaldosteronism type II caused by a novel mutation in WNK4 gene," Endocrine, vol. 33, no. 3, pp. 230-234, 2008.

[16] S. Tsuji, M. Yamashita, G. Unishi et al., "A young child with pseudohypoaldosteronism type II by a mutation of Cullin 3," BMC Nephrology, vol. 14, p. 166, 2013.

[17] A. M. Brooks, M. Owens, J. A. Sayer, M. Salzmann, S. Ellard, and B. Vaidya, "Pseudohypoaldosteronism type 2 presenting with hypertension and hyperkalaemia due to a novel mutation 
in the WNK4 gene," QJM: An International Journal of Medicine, vol. 105, no. 8, pp. 791-794, 2012.

[18] Q. Wang, B.-Y. Cao, C. Su, W.-J. Li, and C.-X. Gong, "Pseudohypoaldosteronism type II caused by CUL3 mutation presented with visual impairment," Chinese Medical Journal, vol. 131, no. 15, pp. 1879-1881, 2018.

[19] T. Sakoh, A. Sekine, T. Mori et al., "A familial case of pseudohypoaldosteronism type II (PHA2) with a novel mutation $(\mathrm{D} 564 \mathrm{~N})$ in the acidic motif in WNK4," Molecular Genetics \& Genomic Medicine, vol. 7, no. 6, p. e705, 2019.

[20] H. Mayan, G. Munter, M. Shaharabany et al., "Hypercalciuria in familial hyperkalemia and hypertension accompanies hyperkalemia and precedes hypertension: description of a large family with the Q565E WNK4 mutation," The Journal of Clinical Endocrinology \& Metabolism, vol. 89, no. 8, pp. 4025-4030, 2004. 